\title{
Reconstruction of Car-Electric Bicycle Side Collision Based on PC-Crash
}

\author{
Shenchao Wang, Yubin Qian, Xianguo Qu \\ College of Automotive Engineering, Shanghai University of Engineering Science, Shanghai, China \\ Email: qianyb@sues.edu.cn
}

Received 2 September 2014; revised 29 September 2014; accepted 21 October 2014

Copyright (C) 2014 by authors and Scientific Research Publishing Inc.

This work is licensed under the Creative Commons Attribution International License (CC BY). http://creativecommons.org/licenses/by/4.0/

(c) (i) Open Access

\begin{abstract}
A collision model, an automobile model and a multi-rigid-body model in PC-crash are analyzed. By simulating a side collision accident between a car and an electric bicycle (EB), a method that reproduces the car-electric bicycle side collision accident based on PC-crash is presented according to some important information such as the final position, the contacting location between the cyclist and the car. A result is obtained by comparing with the reproduced result, the deformation of accident vehicle and the surveillance video. The rollover direction and fells-over sliding direction after the collision and the contacting location and wrap motion of cyclist and electric bicycle are fitting in with the real situation better compared with the reconstruction result in PC-crash. Then the responsive-surface method and MONTEKARLO method are used in MATLAB to analyze the uncertainty of the vehicle speed in reproduced scene results. And the range of values of the pre-collision speed is obtained which makes the reproduced result more objective and convincible that could provide the basis of the accident assessment.
\end{abstract}

\section{Keywords}

PC-Crash, Accident Reproduced, Electric Bicycles, Responsive-Surface, the Analysis of the Uncertainty

\section{Introduction}

With the rapid increase of cars and EBs in domestic land, the side-collision accidents happen more and more between the cars and EBs that the rate in all the city traffic accident is also higher than before [1]. Due to the complexity in collision process, the computer simulation is used which can help to recreate the accident process and to evaluate the accident rapidly that researchers increasingly pay a high attention to. PC-crash is a software extensively used in accident-reproduced based on the applications of the dynamics and kinematics among all the 
reproduced software, such as SMAC, CRASH, EDCRASH, EDSMAC, PC-crash and J2DACS, which relate to the personnel outside the vehicle both at home and abroad [2]. The multi-rigid body model is introduced into the PC-crash to recreate the process of the personnel outside the car and its validation is verified [3]. The accident reproduced is mainly to obtain the participant's speed and collision point at the time of the accident, so that the reference and basis are presented for the accident assessment and liability affirmation. Referring to the reproduced method of car-human collision [4]-[7], the information like the speed of during and before the collision is obtained by reproducing the accident scene, which based on the PC-crash provides the basis for such class of accidents.

\section{Car Models, Model of EB and Cyclists}

A single rigid model is used in vehicle in PC-crash (Figure 1). The shaping parameters can adjust according to the size of actual accident vehicle. The frontal curve outline of the vehicle have a high influence on the motion status after the collision [8], so the simulation result can be more fulfill the actual situation if a 3D DXF (Drawing Exchange Format ) (Figure 2) outline which with an identical and similar shape compared to the actual vehicle is loaded on the outside of the vehicle.

The dummy model can be divided into the multi-rigid dummy, finite dummy, multi-rigid finite dummy [9] in computer simulation. The multi-rigid (Figure 3) dummy is used in PC-crash. Multi-rigid model is consisted of

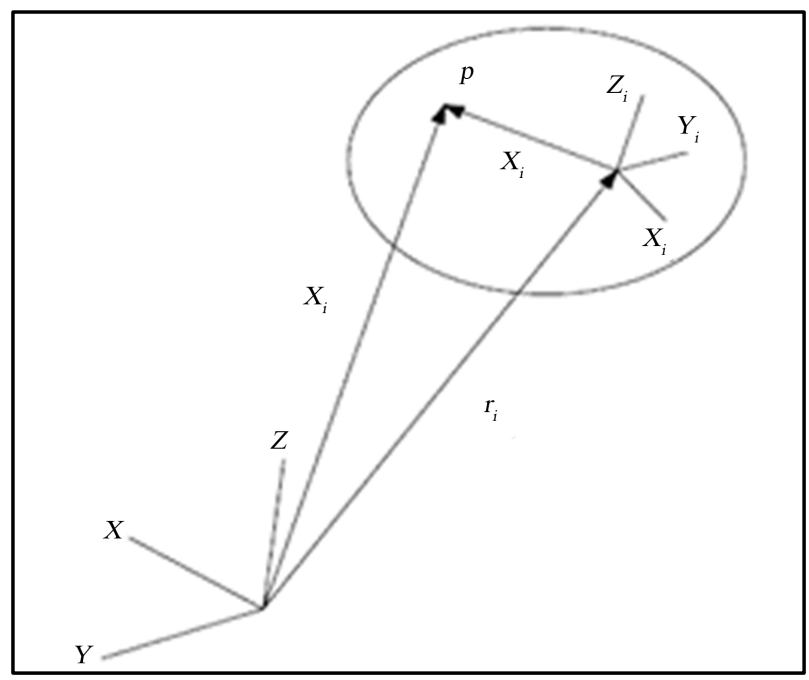

Figure 1. Single rigid model.
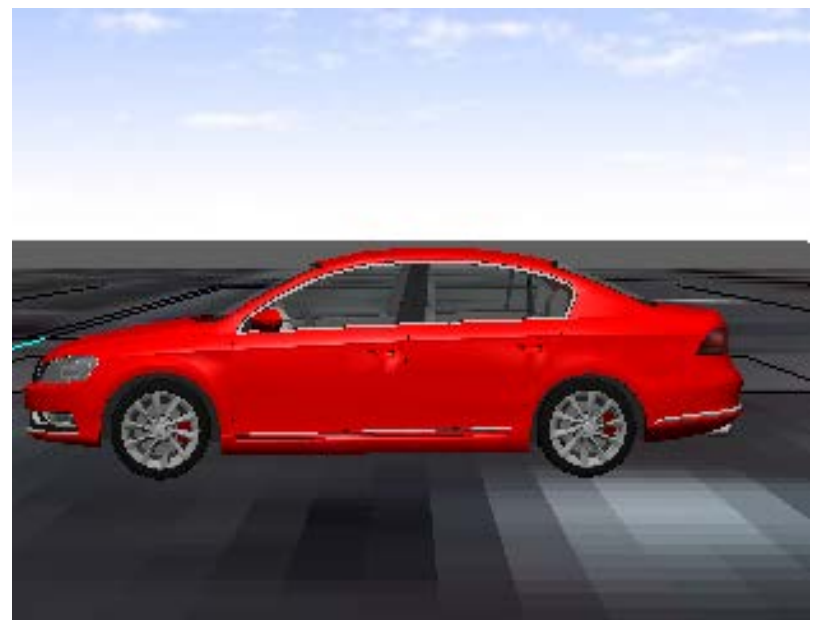

Figure 2. 3D DXF car model. 
some rigid body. Each part of the body is represent the different part of human body such as head, pelvis. All the rigid bodies are connected by the joint. Each rigid body has some characterized parameters like the geometric dimensioning, mass, collision rigid and friction coefficient which all can be set according to the actual measure data in the software. The geometric shape of these parts is set by defining an ellipsoid with an $n$ degree of freedom [10]. Coupling the multi-rigid dummy model with EB model into a Multi-body system in order to participate the reproduced accident scene (Figure 4).

\section{Collision Model}

Car-EB side collision process is complex that mutual dynamic response is existed between the car, cyclist and electric bicycle. The process includes the response between EB and cyclist, the response between EB and car, the response of car and cyclist. The intrusion occurred among these 3 aspects, and the collision force is calculated by a linear rigid function. The force, because of intrusion, can come down to force between Ellipsoid to Ellipsoid contact (Figure 5) and Ellipsoid plane contact (Figure 6). The elasticity of the collision objectives are defined by the recovery coefficient. The friction force is calculated by the normal force when the normal collision force is assured [3].

$$
\begin{aligned}
& \vec{F}_{\text {napproaching }}=\lambda \cdot S \\
& \vec{F}_{\text {nseparating }}=\varepsilon \cdot \lambda \cdot S
\end{aligned}
$$

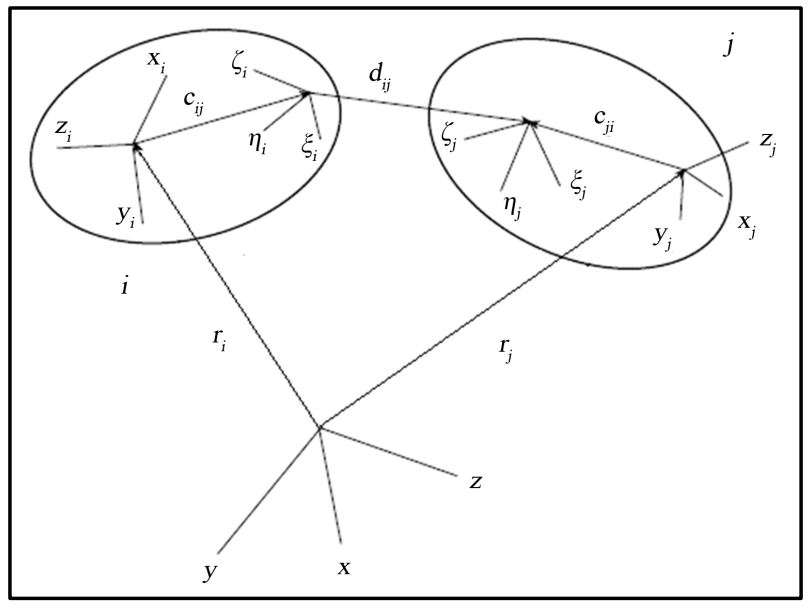

Figure 3. Multi-rigid model.

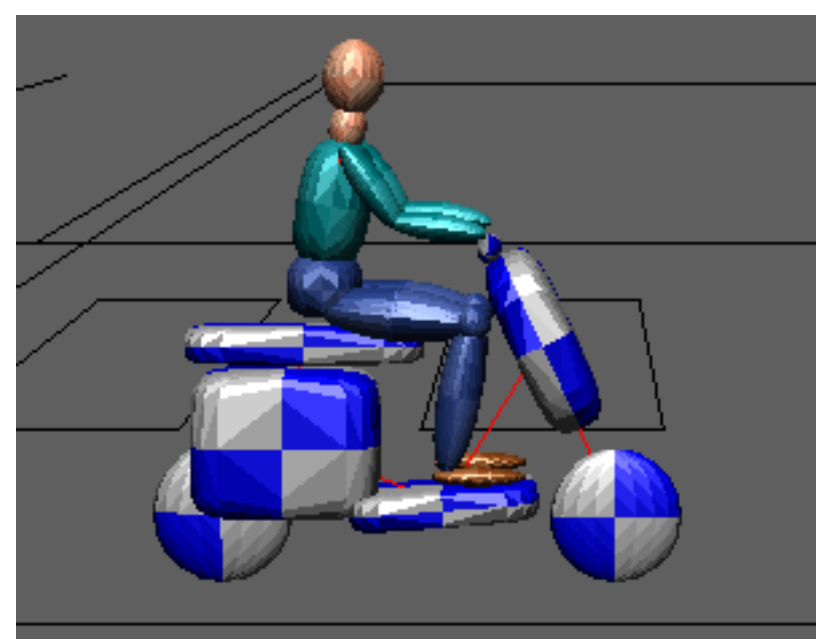

Figure 4. Multi-body model. 


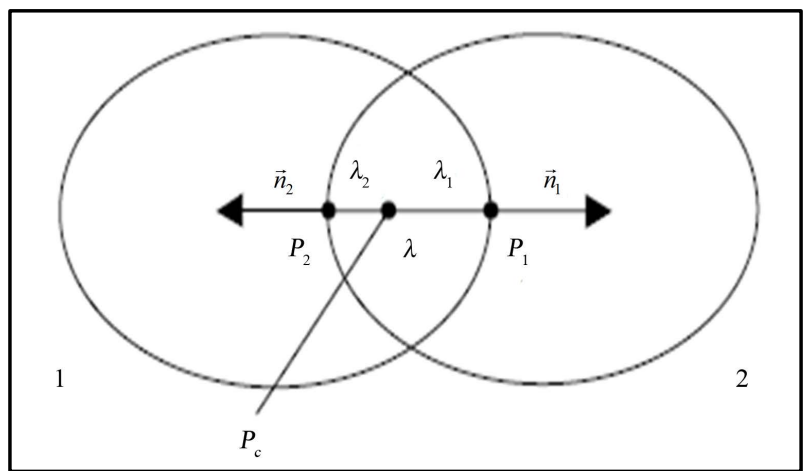

Figure 5. Ellipsoid to ellipsoid contact.

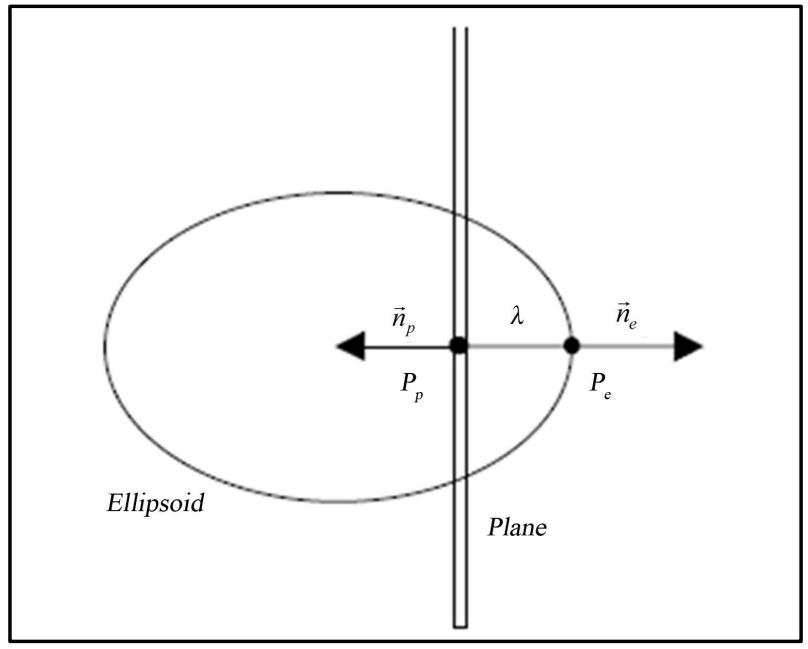

Figure 6. Ellipsoid plane contact.

where:

$\vec{F}_{\text {napproaching }}$ : contact normal force if bodies are approaching;

$\vec{F}_{\text {nseparating }}$ : contact normal force if bodies are separating;

$S$ : stiffness coefficient;

$\lambda$ : penetration depth;

$\varepsilon:$ coefficient of restitution.

$$
\begin{gathered}
\overrightarrow{n_{1}}=-\overrightarrow{n_{2}} \\
\lambda=\left|\mathrm{P}_{1}-\mathrm{P}_{2}\right|=\lambda_{1}+\lambda_{2} \\
\lambda \rightarrow \min \\
\vec{F}_{n 1}=-\vec{F}_{n 2} \\
\vec{F}_{n 1}=-\lambda_{1} \cdot S_{1} \cdot \overrightarrow{n_{1}} \\
\vec{F}_{n 2}=-\lambda_{1} \cdot S_{2} \cdot \overrightarrow{n_{2}} \\
P_{c}=\mathrm{P}_{1}-\overrightarrow{n_{1}} \cdot \lambda_{1}=\mathrm{P}_{1}-\overrightarrow{n_{2}} \cdot \lambda_{2} \\
\mu_{c}=\min \left(\mu_{1}, \mu_{2}\right) \\
\vec{F}_{t 1}=\left|\vec{F}_{n 1}\right| \cdot \mu_{c} \cdot \vec{v}_{P c 2}-\vec{v}_{P c 1}
\end{gathered}
$$




$$
\vec{F}_{t 2}=\left|\vec{F}_{n 2}\right| \cdot \mu_{c} \cdot \vec{v}_{P c 1}-\vec{v}_{P c 2}
$$

where:

$\vec{F}_{n 1}, \vec{F}_{n 2}$ : normal contact force components onto Ellipsoids 1 and 2;

$\vec{F}_{t 1}, \vec{F}_{t 2}$ : tangential contact force components onto Ellipsoids 1 and 2;

$\mu_{c}:$ contact friction.

$$
\begin{gathered}
\overrightarrow{n_{e}}=-\vec{n}_{p} \\
\lambda=\left|\mathrm{P}_{e}-\mathrm{P}_{p}\right| \\
\vec{F}_{n p}=-\vec{F}_{n e} \\
\vec{F}_{n e}=-\lambda_{1} \cdot S_{e} \cdot \vec{n}_{e} \\
\vec{F}_{t e}=\left|\vec{F}_{n e}\right| \cdot \mu \cdot \vec{v}_{P c p}-\vec{v}_{P c e} \\
\vec{F}_{t p}=\left|\vec{F}_{n p}\right| \cdot \mu \cdot \vec{v}_{P c p}-\vec{v}_{P c e}
\end{gathered}
$$

where:

$\vec{F}_{n e}, \vec{F}_{n p}$ : normal contact force components onto ellipsoid and plane;

$\vec{F}_{t e}, \vec{F}_{t p}:$ tangential contact force components onto ellipsoid and plane;

$\mu$ : contact friction.

\section{Case Analysis}

2010-3, moderate rain, bituminous pavement, a car is collide with An EB in the frontal. EB touch down the ground on the left side and stop on the left side of the car about 37.4 meters away. The pedestrian's chest and head are injured and stop at the right side of the car. More information was shown in Table 1.

\subsection{The Process of the Reproduced Accidents}

1) Building the accident scene: as shown in Figure 7, the 2-dimention accident scene is draw in CAD (Computer Aided Design) using the final position of the vehicle, the wheel track as such information according to the accident depth collection data and police spot map. Then the drawing is imported into PC-crash with a DXF format. A 2-dimension accident scene that combines the DXF document with GOOGLE map is built that modify the imported spot scene in PC-crash in order to obtain a better fitment between the spot scene and actual road, as shown in Figure 8.

2) Settings of the model parameters: the VW-PASSAT 1.8 is selected in DSD (Dr. Steffan Datentechnik $\mathrm{GmbH}$ ) database according to the accident depth collection data .Multi-rigid model VESPA ET4 + DRIVER 010910 is selected using on the EB and pedestrian model .It is the moderate rain when the accident happened and has more water on the road. The road friction coefficient is 0.6 , the collision recovery coefficient is 0.1 , and the initial velocity settings of the car and EB are respectively $45 \mathrm{~km} / \mathrm{h}$ and $20 \mathrm{~km} / \mathrm{h}$.

3) The output of the reproduced results: it can be considerable to meet the requirement that the contacting location between the car and pedestrian, the final positions of two vehicles are fit in the actual situation by constantly adjusting the initial collision location and the velocity of the car and EB. The reproduced result basically meets the requirement when the car and EB's velocity are respectively $55 \mathrm{~km} / \mathrm{h}$ and $17 \mathrm{~km} / \mathrm{h}$. The motion process of each participant in reproduced result has been shown in Figure 9 and the accelerate curve of the cyclist's hip and head has shown in Figure 10 and Figure 11.

\subsection{The Analysis of the Reproduced Result}

To analyze the reproduced result combine with the damage deformation of the collision: the dent on the left front of the engine hood is due to the collision between cyclist's hip and the hood which correspond to the first acceleration peak as shown in Figure 10. The net fracture on the upper right of the front windshield is due to the collision between the cyclist's head and the front windshield which correspond to the first acceleration peak as shown in Figure 11. 
Table 1. Case.
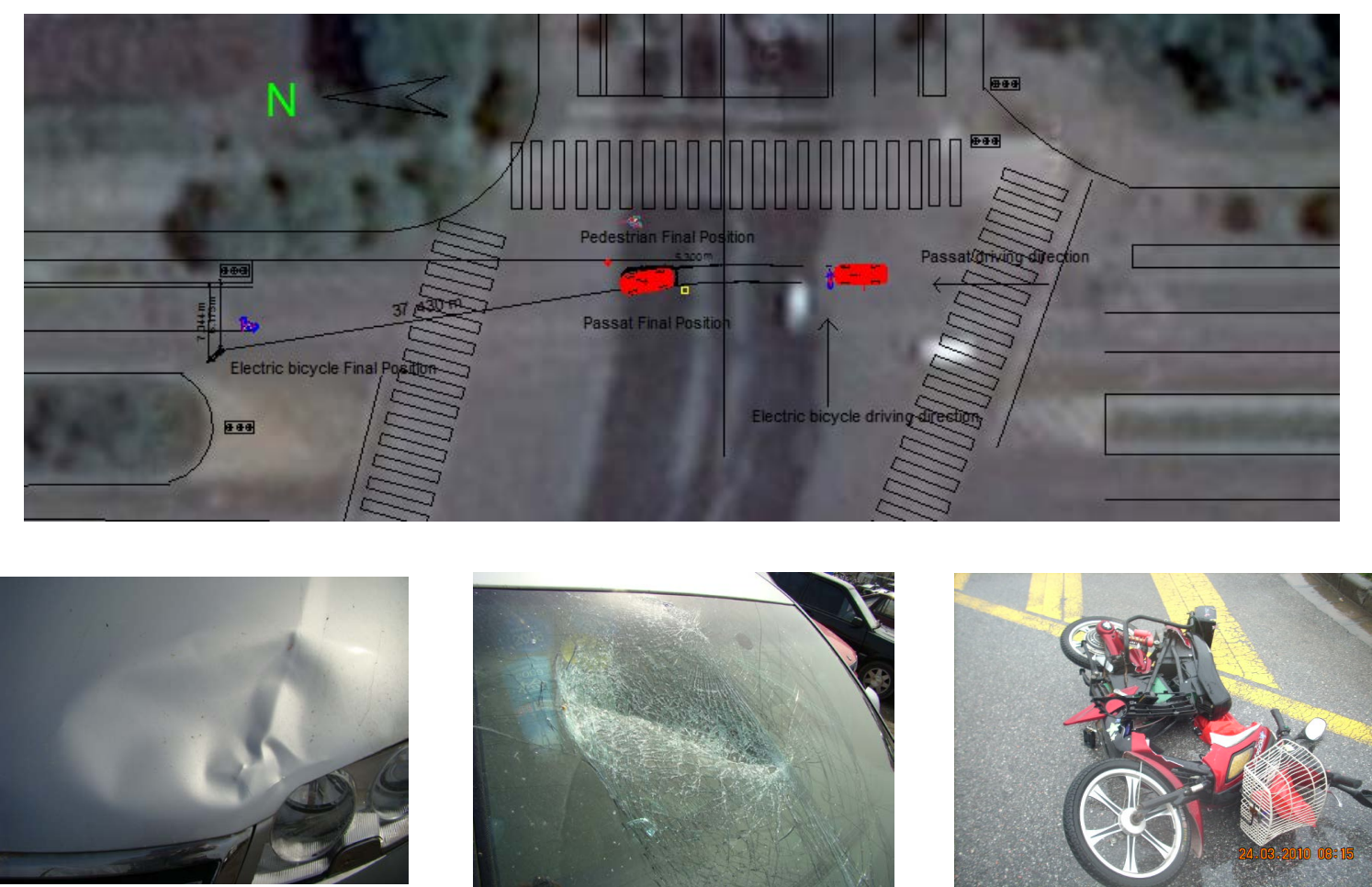

Human

Car

Electric Bicycle

Driver: the speed of the vehicle is 40 - 50 Made in 2008, curb weight is 1552 Curb weight is $104 \mathrm{~kg}$, $\mathrm{km} / \mathrm{h}$ before the accident. Urgent braking $\mathrm{kg}$, dent deformation in front left system is used after the impact.Cyclist: side of the engine hood, mesh male, age of 47, wearing the helmet, right crumbles in upper front side of side of hip and head are injured. crumble in the middle of the distance between the rear right side, and apparent frictional trace in outer edge of left side. wheel of EB and the fron

wheel of PASSAT is 37.4 meters.

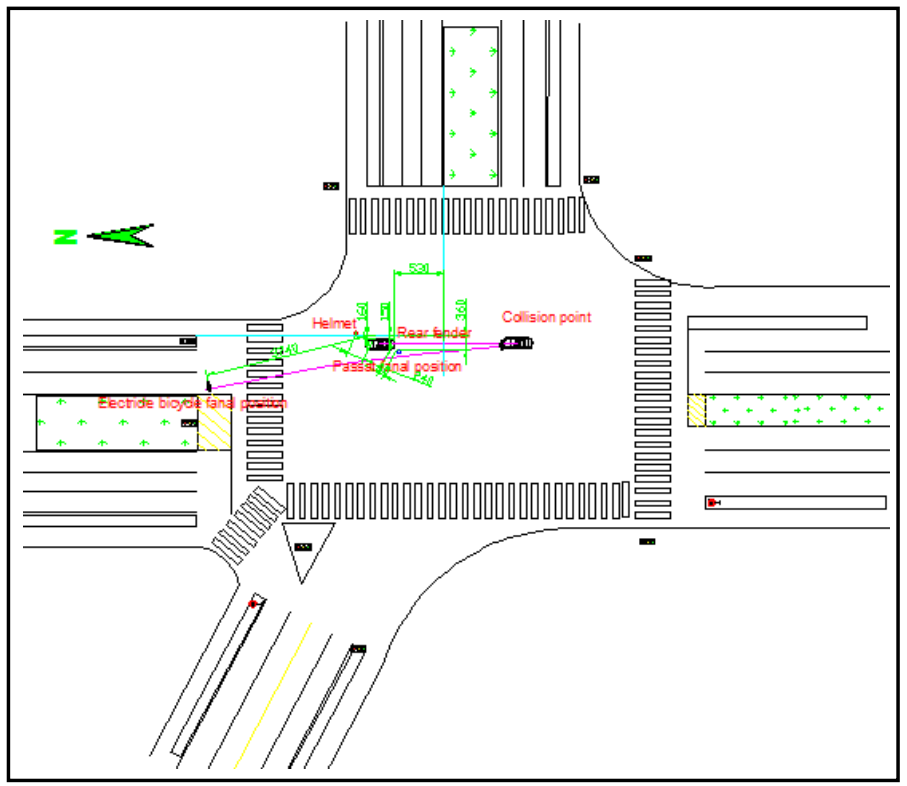

Figure 7. DXF accident scene. 


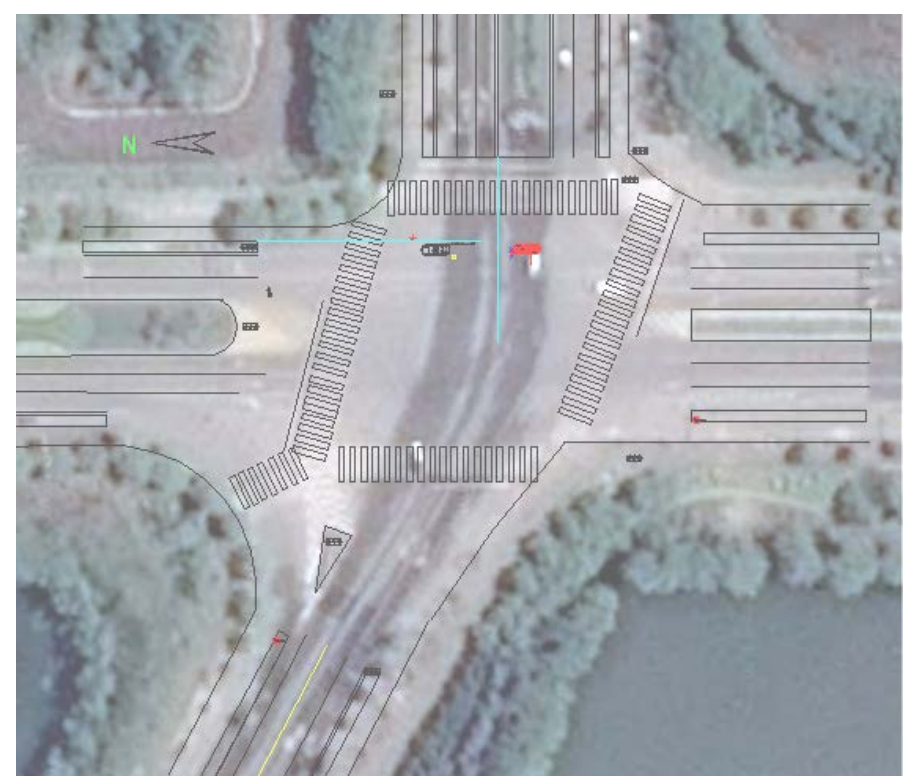

Figure 8. Google map with DXF accident scene.

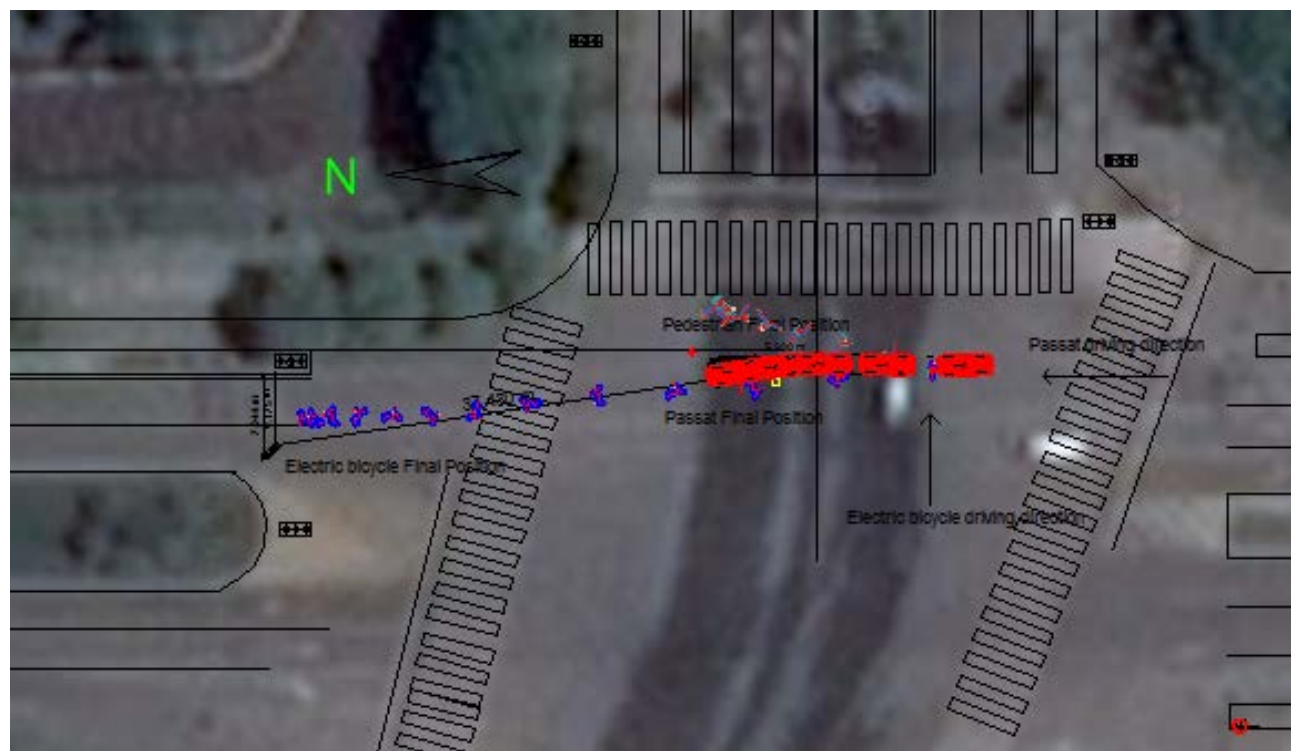

Figure 9. Motion process.

To analyze the reproduced result combine with the surveillance video: the right side of EB collide with frontal side of the car where about 1/2 of the car's width. EB follows the car sliding some distance then hit the ground at its left side after the collision and finally stops about 34 meters away the front left side of the car, 3 meters' deviation from actual situation. The motion process and the video information are basically consilient. The right side of cyclist's hip is firstly collide with front left side of the engine hood, rolling after the body upspring, then the cyclist's head and shoulder collide with the upper side of the front windshield, separate from the car finally, and land up the right side of the car. The cyclist stops about 5 meters from the right side of the car which's motion process is correspond to the video information. The comparison of video and reproduced result has been shown in Figure 12.

\subsection{The Analysis on the Uncertainty of the Reproduced Result}

The analysis on the uncertainty of the reproduced result is an important part of the reproduced result. Under the 


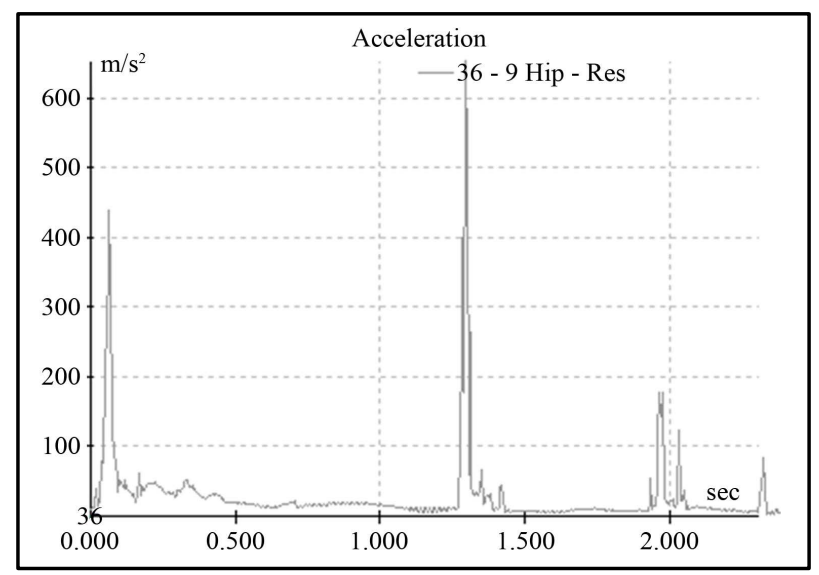

Figure 10. Hip accelerate curve.

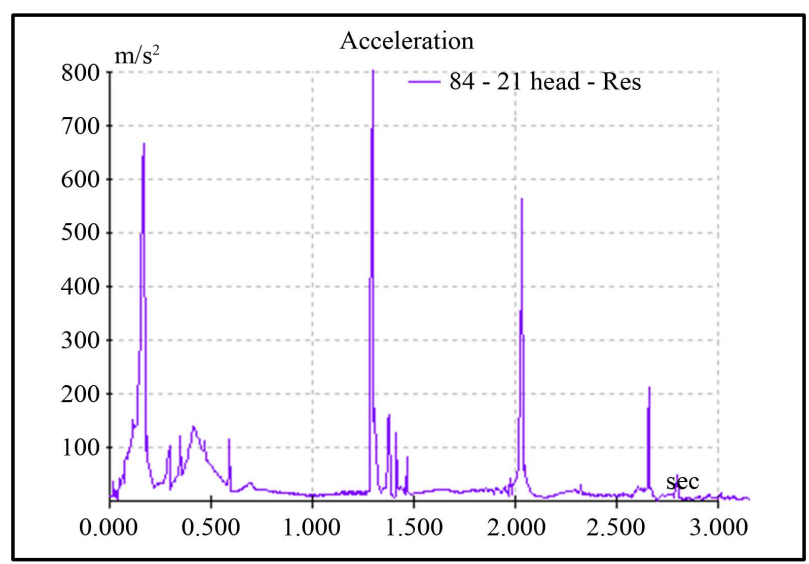

Figure 11. Head accelerate curve.

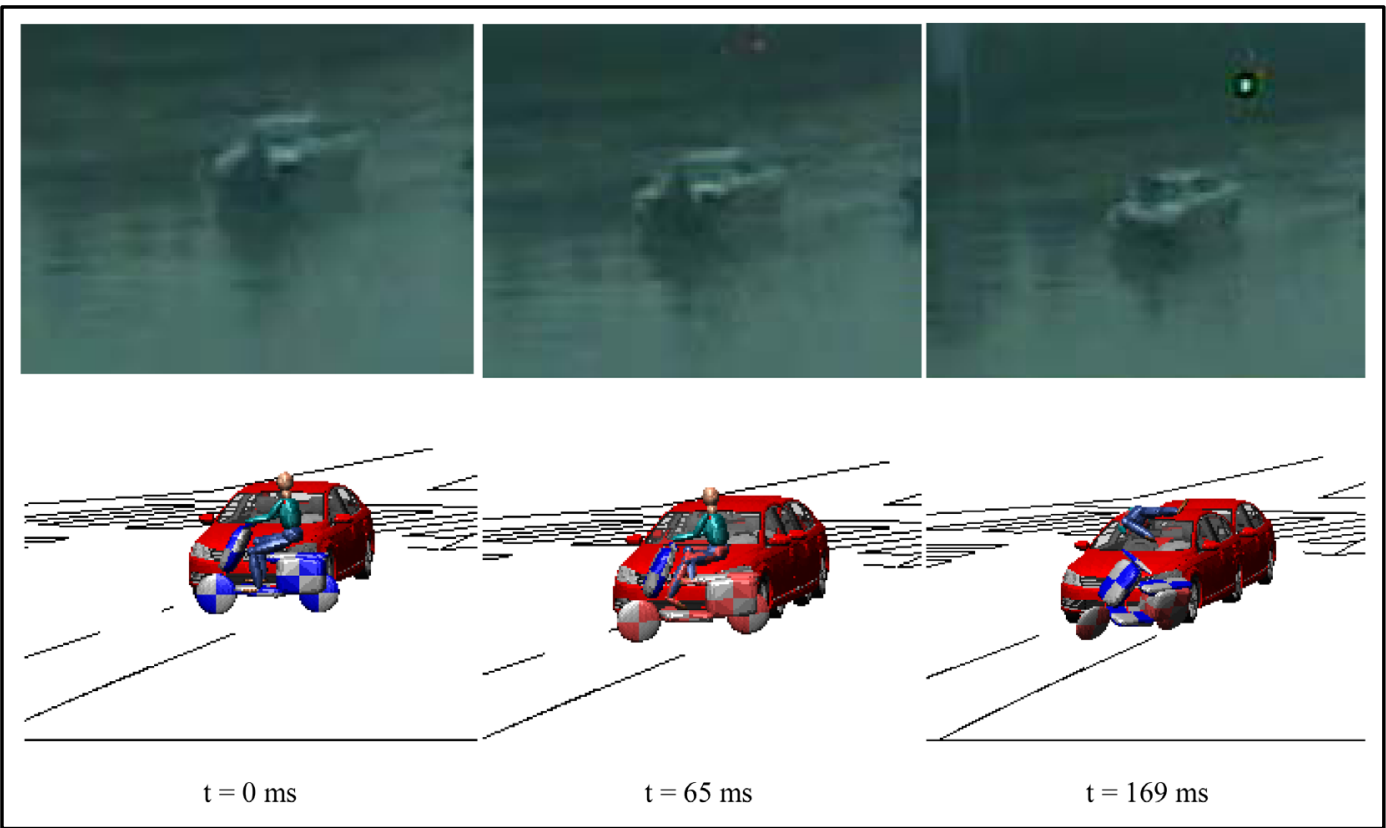

Figure 12. Comparison of video and reproduced result. 
circumstances of knowing some value range, the reproduced result is more objective and can provide more basis on accident liability identification. The model display expression in PC-crash is unknown [11]. But the similar expression can be obtained through the curve method. Then the uncertainty of the reproduced result is analyzed using this similar expression and the MONTECARO method.

Analyzing the parameters' weight in PC-crash [12] and pick $t$ as the braking coordinate time, $S$ as the distance from the collision point to final location, $\mathrm{U}$ as the friction coefficient of car and ground. All the parameters can be the uncertainty input parameters as the reproduced result of uncertainty analysis of the velocity $\mathrm{V}$. In this case $t \in[0.1,0.3] \mathrm{s}$, if these 3 parameters are normal distribution, the experiment samples are generated by using the Latin Hypercube Sampling (LHS) [13] in tool box in MATLAB. The experiment must strictly follow the experiment (Table 2).

The formula is obtained by regression analysis in MATLAB using the data in Table 2. The correlation coefficient is 0.9648 and the residuals standard deviation is 0.5183 .

$$
v=-6.6734 t+10.9117 S+112.6442 u+6.6527 t^{2}-0.2421 S^{2}-71.1539 u^{2}
$$

Using the relevant surface to make the MONTECARLO samples are $10^{6}$ [14]. Then the value range of the car velocity from the driver is improper. So the velocity $40-50 \mathrm{~km} / \mathrm{h}$ of the record of the car driver is not correct. This given velocity range is objective and reasonable in the liability identification.

\section{Conclusions}

A multi-rigid model and a collision model in PC-crash are analyzed. Then an accident reproduced method is presented based on PC-crash combined with a side collision between a car and an EB. The follow conclusions are obtained by verifying the reproduced result and its uncertainty:

1) 2-dimension accident scene can be built rapidly and correctly in PC-crash combining the Google map and actual situation of scene road. Not only both the participants' information is accurately determined, but also the route information in accidental site is reflected. It is the basis of the reason of accident occurring.

2) The rollover direction after the collision and sliding direction after hitting the ground of the EB are fitting in with the actual situation during the PC-crash reproduced collision, so is the contacting location between the cyclist and wrap motion. The cyclist can easily fly over the car roof after colliding with the front windshield which is not fulfilling the actual situation and is more similar to human-car collision situation.

3) The value range is obtained by analyzing the uncertainty of the velocity of the car in reproduced result which is more objective and convincible. This method also can be used in the uncertainty analysis of the EB velocity and other reproduced results. The regularities of the distribution of 3 input parameters, braking coordinate time $t$, the distance from the collision point to the final position $S$ and friction coefficient $u$ are assumed uniform distribution so that the accurate distribution situation still needs further discussion.

\begin{tabular}{ccccc}
\hline \multicolumn{5}{l}{ Table 2. Table and result of test. } \\
\hline Number & $\boldsymbol{t} / \boldsymbol{s}$ & $\boldsymbol{S} / \mathbf{m}$ & $\boldsymbol{u}$ & $\boldsymbol{v} /(\mathbf{k m} / \mathbf{h})$ \\
\hline 1 & 0.28 & 19.22 & 0.64 & 57.5 \\
2 & 0.14 & 20.3 & 0.5 & 54.3 \\
3 & 0.2 & 17.6 & 0.58 & 52.7 \\
4 & 0.26 & 18.14 & 0.56 & 52.67 \\
5 & 0.16 & 21.38 & 0.52 & 57 \\
6 & 0.2 & 20.3 & 0.6 & 57.7 \\
7 & 0.22 & 18.68 & 0.66 & 57.55 \\
8 & 0.12 & 21.92 & 0.62 & 59.4 \\
9 & 0.18 & 22.46 & 0.6 & 59.4 \\
10 & 0.24 & 19.76 & 0.68 & 58.5 \\
11 & 0.1 & 20.84 & 0.54 & 57.5 \\
\hline
\end{tabular}




\section{Acknowledgements}

This work was supported by China National Automobile Accident In-Depth Investigation System (NAIS) and Shanghai University of Engineering Science Traffic Safety Analysis Center.

\section{References}

[1] Wang, M.L. (2010) Safety Analysis of Electric Bicycle on Urban Road. Southwest Jiaotong University, Chengdu.

[2] Yuan, Q. and Li, Y.B. (2002) Review and Trend of the Technology of Vehicle Crash Reconstruction. Journal of Highway and Transportation Research and Development, 2, 122-125.

[3] Moser, A., Steffan, H., et al. (1999) The Pedestrian Model in PC-Crash-The Introduction of a Multi Body System and Its Validation. SAE Paper, 794-802.

[4] Zou, T.F., Yu, Z. and Chai, M. (2010) Study on Laws of Vehicle-Pedestrian Accident Simulation Based on Pc-Crash Software. Journal of China Safety Science, 2, 54-58.

[5] Lin, Q.F. and Xu, H.G. (2007) Simulation Model for High Speed Car-Pedestrian Collision Based on PC-Crash. Automotive Engineering, 7, 562-565.

[6] Zou, T.F., Yu, Z., Chai, M. and Liu, J.K. (2011) Car-Pedestrian Accident Reconstruction Based on Pc-Crash. Journal of Vibration and Shock, 3, 215-219.

[7] Wang, G.L., Lu, Y. and Zou, K.K. (2008) Study of Simulation of Vehicle-Pedestrian Collision Accident. Tractor and Farm Transporter, 1, 30-31.

[8] Steffan, H. (2006) Pc-Crash Technical Manual Version 7.3. Dr. Steffan Datentechnik GmbH, Linz.

[9] Guo, L. (2008) Simulation Research on Collision Accidents between Vehicle and Two-Wheelers and Its Application. Shanghai Jiao Tong University, Shanghai.

[10] Moser, A., Steffan, H., et al. (2000) Validation of the Pc-Crash Pedestrian Model. SAE Paper, 1336-1339.

[11] Zou, T.F., Yu, Z., Chai, M., et al. (2010) Application of Response Surface Method to Uncertainty Analysis on Accident Reconstruction Results. Automotive Engineering, 32, 116-118.

[12] Wang, H.Y. and Shao, W.Y. (2009) Error Analysis of Traffic Accident Reconstruction Based on PC-Crash. Journal of Tongji University (Natural Science), 4, 531-536.

[13] Zou, T.F., Chai, M., Liu, J.K. and Li, Y.L. (2013) Method for Analyzing Uncertainty of Simulation Results in Accident Reconstruction. Journal of System Simulation, 5, 911-914.

[14] Zou, T.F. and Zhao, L.X. (2013) A Method for Estimating Sample Size of Monte Carlo Method in Accident Reconstruction. Journal of China Safety Science, 5, 22-26. 
Scientific Research Publishing (SCIRP) is one of the largest Open Access journal publishers. It is currently publishing more than 200 open access, online, peer-reviewed journals covering a wide range of academic disciplines. SCIRP serves the worldwide academic communities and contributes to the progress and application of science with its publication.

Other selected journals from SCIRP are listed as below. Submit your manuscript to us via either submit@scirp.org or Online Submission Portal.
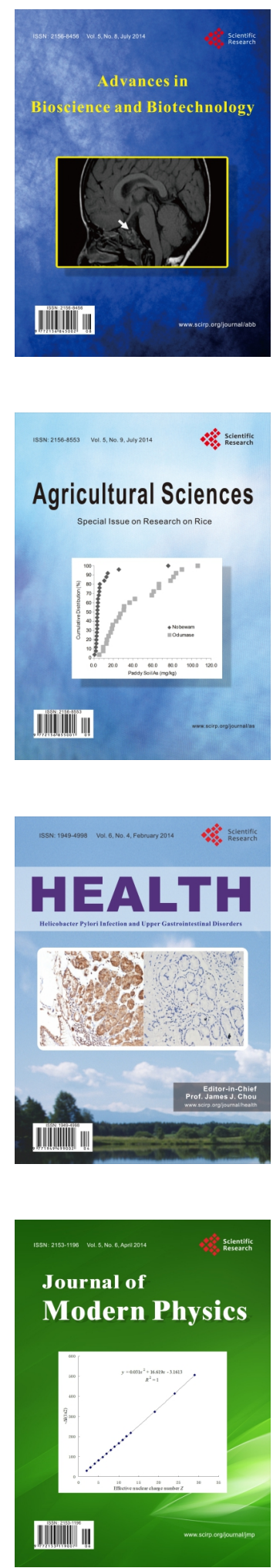
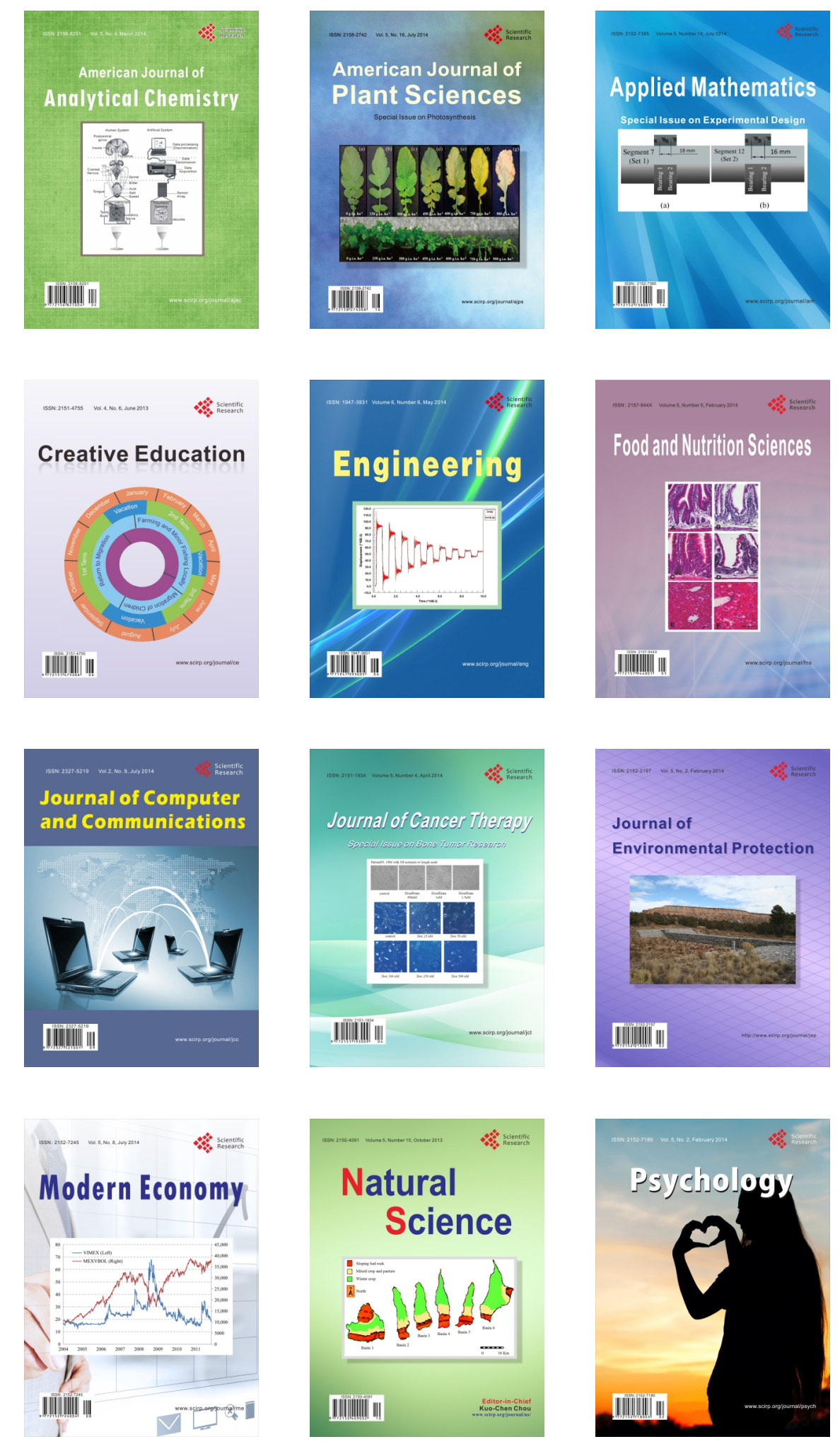